\title{
Investigation of a One-Time Phenol Application for Pilonidal Disease
}

\author{
Cuneyt Kayaalp ${ }^{\mathrm{a}} \quad$ Aydemir Olmez $^{\mathrm{b}} \quad$ Cemalettin Aydin $^{\mathrm{a}} \quad$ Turgut Piskin $^{\mathrm{a}}$ \\ Latif Kahraman ${ }^{c}$ \\ Department of General Surgery, ${ }^{\mathrm{a}}$ Inonu University, ${ }^{\mathrm{b}}$ Beydagi State Hospital, and 'Darende State Hospital, \\ Malatya, Turkey
}

\section{Key Words}

Pilonidal disease $\cdot$ Phenol $\cdot$ Outpatient surgery $\cdot$

Postoperative care $\cdot$ Time off work

\begin{abstract}
Objective: To investigate wound healing rates and postoperative recovery of patients after a one-time phenol application for pilonidal disease. Subjects and Methods: A total 30 consecutive patients with chronic pilonidal disease ranging from midline to complex sinuses were enrolled in the study. No preoperative laboratory examinations or bowel preparation were required. No antibiotic prophylaxis or sedation was used. A small incision was made on the midline and hair/ debris in the sinuses was removed. A cotton swab with saturated phenol was moved into the cavity and the phenol was left for $2 \mathrm{~min}$. No special dressing was necessary and patients left the hospital immediately afterwards. Patients filled out a daily questionnaire for 7 days. We did not intervene in the wounds with a second phenol application or curettage during the observation period. Wounds were inspected at weekly intervals for 2 months. Results: At the end of the third day, $97 \%$ of the patients were pain-free and $100 \%$ of the patients were free from analgesics. Time off work was 2 days for most patients (93.3\%). Twenty-eight (93.3\%) patients were satisfied with the procedure, they found it easy and
\end{abstract}

painless and suggested the procedure to other patients. Twenty-five (83\%) patients were asymptomatic at the end of 2 months' observation and the remaining 5 patients had unhealed sinuses. Mean time for wound healing was 25 days (range 10-63 days). There were 4 recurrences after a mean of 14 months' follow-up and the overall success rate was $70 \%$. Conclusions: A one-time phenol application was an effective treatment for pilonidal disease with acceptable wound healing rates, less postoperative pain and less time off work. Hence it can be an alternative treatment modality.

Copyright $\odot 2010$ S. Karger AG, Basel

\section{Introduction}

The ideal treatment of pilonidal disease should be simple, cost-effective, preferably be done in outpatient clinics under local anesthesia, should cause minimal discomfort and time off work, and have a low recurrence rate. Surgery is a well-described treatment for sacrococcygeal pilonidal disease and surgical methods usually include excision of sinus tracts followed by primary midline closure, flap closure or leaving the wound open to heal by secondary intention [1]. Surgical excision methods are still far from being defined as ideal treatments. They are usually performed under regional or general anesthesia,

\section{KARGER}

Fax +4161306 1234

E-Mail karger@karger.ch

www.karger.com
(C) 2010 S. Karger AG, Basel

$1011-7571 / 10 / 0193-0212 \$ 26.00 / 0$

Accessible online at:

www.karger.com/mpp
Cuneyt Kayaalp, MD, Associate Professor

Department of General Surgery, Turgut Ozal Medical Center, Inonu University TR-44280 Malatya (Turkey)

Tel. +90 422341 0660/3706, Fax +90 4223410229

E-Mail cuneytkayaalp@ hotmail.com 
require hospital stay, and cause considerable loss of working time.

Phenol treatment for pilonidal disease was first described by Maurice and Greenwood [2]. This method avoids excision of the sinus and is based on the destruction of the pathologic epithelium of the sinus. Pioneers of the method thought that if the epithelium of the tract could be destroyed, any infection present sterilized and embedded hair removed, there should be no reason why the sinus should not heal [2]. Previous studies of phenol treatment for pilonidal disease did not mention specifically the natural course of the wound after one application or postoperative recovery of the patients [3-9]. In the present study we investigated the efficacy of a one-time phenol treatment of pilonidal disease.

\section{Subjects and Methods}

Between June and September 2007, a total of 30 consecutive patients with chronic pilonidal disease received phenol treatment. The cases ranged from simple chronic midline sinuses to complex sinuses extending to the lateral gluteal region. However, cases of acute abscess were excluded. Patients were informed about this alternative procedure and procedures were done at an outpatient clinic. Most patients were healthy young men and no laboratory examinations were required before the procedure. No bowel preparation and no antibiotic prophylaxis were used. Patients were prone during the procedure and sedation was not used. Buttocks were shaved just before the procedure and skin was cleaned with an antiseptic solution (Batticon). Local anesthesia including lidocain $(20 \mathrm{mg} / \mathrm{ml})$ and epinephrine $(0.0125 \mathrm{mg} / \mathrm{ml})$ (Jetokain, Adeka Ilac Sanayi, Istanbul) were injected slowly into the midline subcutaneous skin around the orifices. A small incision was made on the midline including the openings to communicate them. Minimal bleeding was stopped using electrocautery. Hairs and debris in the sinuses were removed by curettage. Dimensions of the sinus cavity were measured. The preferred phenol was liquid, $80 \%$ in concentration, and a cotton swab saturated with phenol was moved into the cavity which was followed by changing of the cavity color to whitish. The phenol was left for $2 \mathrm{~min}$ and at the end, the excess phenol was mopped away with the debris. No special dressing was necessary and a light dressing of gauze was sufficient. The patients were able to leave the hospital immediately after the procedure. It was emphasized to patients that they must be meticulous in keeping the area clean and free from hair. Patients were requested to fill a daily questionnaire for 7 days after the procedure (table 1). Wounds were inspected at weekly intervals for 2 months. At the end of 2 months, wounds were evaluated as healed or not. This protocol was adhered to strictly and we did not intervene in the wounds with a second phenol application or curettage during the 2 months. In the long term, patients were followed up by outpatient controls or telephone calls. All the patient demographics, procedure details and follow-up protocols were recorded in a standard form. Patients were followed for a mean of 14 months (range 1316). Descriptive statistics were used for analysis.

One-Time Phenol Application for Pilonidal Disease
Table 1. Questionnaire after single-application phenol treatment

How did you find the procedure? Easy or difficult?

Do you suggest the procedure for anyone else?

Do you feel any pain during your activities today

(for 7 days)?

If yes: do you need any analgesics today (for 7 days)?

When did you begin study again (working, going to

school etc.)?

\section{Results}

There were 24 males and 6 females with a mean age of 24 years (range 16-37). All cases were primary pilonidal disease except 1 . There were midline orifices in 23 patients $(77 \%)$ and the remaining 7 patients had lateral orifices. The number of orifices ranged from 1 to 8 (mean 2.8). Mean vertical length of the sinus cavities was $27 \mathrm{~mm}$ $(10-60 \mathrm{~mm})$, mean width was $13 \mathrm{~mm}(6-40 \mathrm{~mm})$, and the mean length of incision was $12 \mathrm{~mm}(5-35 \mathrm{~mm})$. No patient was excluded from the study once enrolled, and all the procedures were completed without any additional anesthesia or sedation. There were no admissions from the day-case surgery and no unplanned readmissions.

Postoperatively, patients were asked if they suffered from pain and for their requirement for analgesics, each day for 7 days. Patients who reported pain and patients who required analgesics were $10(33 \%)$ and $3(10 \%)$, respectively, on day 2 . On day 3 , only 1 patient suffered pain and required analgesics. After the third day, 97 and $100 \%$ of the patients were free from pain and analgesics, respectively (fig. 1). Time off work was 2 days for 28 (93.3\%) patients and 3 days for the remaining 2 (6.7\%) patients. Twenty-eight (93.3\%) patients were satisfied with the procedure, they found it easy and painless, and they suggested the procedure to other patients. The remaining 2 patients did not find the procedure painful or difficult, but they found it stressful and irritating due to live surgery or surgeon's touch.

There were $4(10 \%)$ complications, 1 was a superficial skin burn due to accidental scattering of phenol, which healed uneventfully within 3 days. The remaining 3 patients had wound abscesses during the healing period due to early closure of the skin and all of them were treated by drainage and antibiotics. One of these patients healed uneventfully but the others resulted in prolonged purulent discharge and were regarded as treatment failures.

At the end of 2 months, 25 (83\%) patients were asymptomatic. The remaining $5(17 \%)$ patients suffered from on- 
Table 2. Results of single-application phenol treatment

\begin{tabular}{lrll}
\hline Author and year & Patients & $\begin{array}{l}\text { Success } \\
\text { rate, \% }\end{array}$ & $\begin{array}{l}\text { Mean follow-up } \\
\text { time }\end{array}$ \\
\hline Schneider et al. [3] & 37 & 60 & $3.5-7.5$ years \\
Vara-Thorbeck et al. [4] & 67 & 78 & $1-3$ years \\
Stansby and Greatorex [5] & 104 & 65 & 8 months \\
Kelly and Graham [6] & 44 & 61 & NA \\
Hegge et al. [7] & 48 & 56 & 3 years \\
Stephens and Sloane [8] & 30 & 83 & $6-36$ months \\
Stewart and Bell [9] & 30 & 63 & 18 months \\
Maurice and Greenwood [2] & 21 & 67 & 18 months \\
This study & 30 & 70 & 14 months \\
\hline
\end{tabular}

$\mathrm{NA}=$ Not available

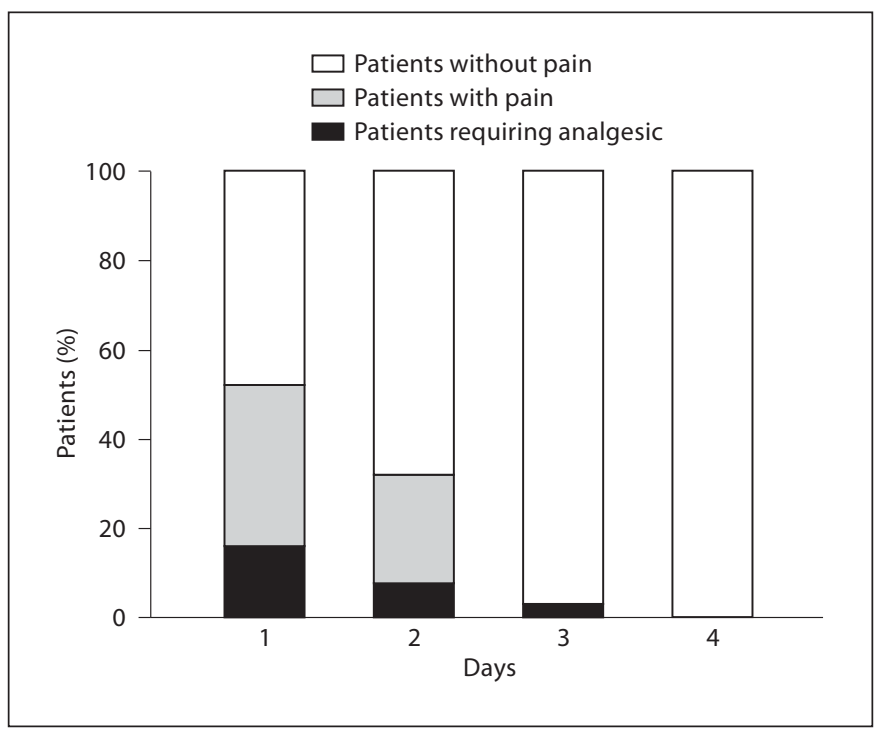

Fig. 1. Postoperative pain and analgesic requirements. failures. The 25 asymptomatic patients were examined for wound healing and 14 (47\%) of them had a complete cure with the closure of the openings, while the remaining 11 (37\%) patients had very small orifices that could not be probed. Mean time for wound healing was 25 days (range 10-63 days).

At the end of the follow-up period there were 4 (16\%) recurrences in the 25 successfully treated patients. These patients were also treated by excision and flap procedures. The overall success rate was 21 of 30 patients $(70 \%$, table 2). The success rate reflected discontinuation of purulent discharge. Four patients with recurrent and 5 with prolonged discharge were treated with excision and flap procedures without morbidity.

\section{Discussion}

There is no consensus on success or failure of phenol treatment for pilonidal disease. Success may be symptomatic relief [10] or anatomically healed sinuses [3]. Symptomatic healing is cessation of symptoms that include pain, swelling, or discharge. Anatomical healing is complete skin epithelialization in addition to symptomatic relief. These distinctions resulted in variable treatment outcomes (table 2). We describe for the first time both symptomatic and anatomic healing in the same study. Our anatomical healing results were only $47 \%$, but overall asymptomatic patients were $70 \%$.

Most studies report repeated applications of phenol in cases of prolonged healing or recurrences $[2-9,11]$. Timing or indications of repeated applications are quite variable. This causes confusion in the success rates as well. We performed only a single application with a strict protocol, and based on 2-month observation we decided whether or not the procedure was successful. We defined recurrence as occurrence of the same complaints after an asymptomatic period. The overall failure rate was a combination of the symptomatic patients and the recurrent patients. Our long-term success rate of $70 \%$ was within $56-83 \%$ of previous studies (table 2).

Before the procedure, most of our patients were anxious about the postoperative course of pilonidal sinus surgery. They had heard a story of difficulty on sitting on the buttocks for days or even weeks after pilonidal sinus surgery. However, most of our patients (93.3\%) were satisfied with the procedure and the degree of pain was far much less than anticipated. Most patients (80\%) did not require analgesics even on postoperative day 1 . Patients had lost minimal time off work and most of them were able to return to their normal routine within 2-3 days. During the healing time, there was usually a little serous discharge from the sinus and this discharge gradually diminished during healing. This soiling did not hinder the daily activities and work of the patients and ordinary sanitary napkins over the wound were enough.

The most common postoperative complications reported after phenol treatment are development of ab- 
scesses and cellulites $[3-6,10]$. They were attributed to leakage of phenol into the surrounding tissues due to either too much pressure at the time of injections, or to opening up a false tract by the preliminary probing. We suggested phenol application with a swab without pressure and without probing. However, we reported 10\% with abscesses after phenol application and we attributed this to the early closure of the skin before healthy granulation of the sinus cavity. The abscesses were all cured by surgical drainage but two of three resulted in prolonged drainage.

\section{Conclusion}

We demonstrated that phenol application was closer to the ideal treatment of pilonidal disease by application under local anesthesia in outpatient clinics. Additionally, it was simple and cost-effective. Although the success rate was not very high (70\%), phenol application was thought to be sufficiently successful to warrant using it as a routine for most cases. We believe that a longer follow-up to estimate the results after 1 year is necessary. At present, we suggest that phenol application is a good alternative for patients who desire less postoperative pain or less time off work and acceptable wound healing rates.

\section{References}

1 McCallum I, King PM, Bruce J: Healing by primary versus secondary intention after surgical treatment for pilonidal sinus. Cochrane Database Syst Rev 2007;4: CD006213.

2 Maurice BA, Greenwood RK: A conservative treatment of pilonidal sinus. Br J Surg 1964; 51:510-512.

-3 Schneider IH, Thaler K, Köckerling F: Treatment of pilonidal sinuses by phenol injections. Int J Colorectal Dis 1994;9:200-202.

4 Vara-Thorbeck R, Mekinassi K, Berchid S: Phenol treatment of pilonidal sinuses. Zentralbl Chir 1990;115:777-780.
5 Stansby G, Greatorex R: Phenol treatment of pilonidal sinuses of the natal cleft. Br J Surg 1989;76:729-730.

6 Kelly SB, Graham WJ: Treatment of pilonidal sinus by phenol injection. Ulster Med J 1989;58:56-59.

7 Hegge HG, Vos GA, Patka P, Hoitsma HF: Treatment of complicated or infected pilonidal sinus disease by local application of phenol. Surgery 1987;102:52-54.
8 Stephens FO, Sloane DR: Conservative management of pilonidal sinus. Surg Gynecol Obstet 1969;129:786-788.

-9 Stewart TJ, Bell M: The treatment of pilonidal sinus by phenol injection. Ulster Med J 1969;38:167-171.

10 Kaymakcioglu N, Yagci G, Simsek A, Unlu A, Tekin OF, Cetiner S, Tufan T: Treatment of pilonidal sinus by phenol application and factors affecting the recurrence. Tech Coloproctol 2005;9:21-24.

- 11 Dogru O, Camci C, Aygen E, Girgin M, Topuz O: Pilonidal sinus treated with crystallized phenol: an eight-year experience. Dis Colon Rectum 2004;47:1934-1938. 